\title{
VIEW CLASSIFICATION OF MEDICAL X-RAY IMAGES USING PNN CLASSIFIER, DECISION TREE ALGORITHM AND SVM CLASSIFIER
}

\author{
Sumathi Ganesan ${ }^{1}$, T.S.Subashini ${ }^{2}$ \\ ${ }^{1}$ Assistant Professor, Department of Computer Science and Engineering, Faculty of Engineering and Technology, \\ Annamalai University, India. \\ ${ }^{2}$ Associate Professor, Department of Computer Science and Engineering, Faculty of Engineering and Technology, \\ Annamalai University, India.
}

\begin{abstract}
:
In this era of electronic advancements in the field of medical image processing, the quantum of medical X-ray images so produced exorbitantly can be effectively addressed by means of automated indexing, comparing, analysing and annotating that will really be pivotal to the radiologists in interpreting and diagnosing the diseases. In order to envisage such an objective, it has been humbly endeavoured in this paper by proposing an efficient methodology that takes care of the view classification of the X-ray images for the automated annotation from their vast database, with which the decision making for the physicians and radiologists becomes simpler despite an immeasurable and ever-growing trends of the X-ray images. In this paper, X-ray images of six different classes namely chest, head, foot, palm, spine and neck have been collected. The framework proposed in this paper involves the following: The images are pre-processed using M3 filter and segmentation by Expectation Maximization (EM) algorithm, followed by feature extraction through Discrete Wavelet Transform. The orientation of X-ray images has been performed in this work by comparing among the Probabilistic Neural Network (PNN), Decision Tree algorithm and Support Vector Machine (SVM), while the PNN yields an accuracy of 75\%, the Decision Tree with 92.77\% and the SVM of 93.33\%.
\end{abstract}

Key Words: M3 filter, Expectation Maximaization, Discrete Wavelet Transformation, Probabilistic Neural Network, Decision Tree Algorithm and Support Vector Machine.

\section{INTRODUCTION}

Orientation of $\mathrm{X}$-ray images is referred as radiographic positioning or view of an X-ray image. Radiographic positioning is highly standardized for better positioning, so as to accurately interpret for the correct diagnosis by the physician and radiologists. Hence, it plays a pivotal role in viewing the particular portions or areas to be examined. Radiographic positions viz. lateral view, oblique view, anterior-posterior view, posterior-anterior view etc., which are all so classified based on the way the X-ray images are radiographed with respect to the object and the film. When the X-rays have passed through the object from front to back of the patients, it is referred as Anterior-Posterior (AP) view. If it is taken from back to front of the patient it is said to be Posterior-Anterior (PA) view. When it is passed through the object from the side of the patients, it is said to be lateral view. In oblique view, X-rays have passed through the object based on the angle. Hence, it is quite inevitable that there needs to be suitable computational algorithms for the detection of the orientation of the X-ray images.

Keeping such objectives in view, an attempt has been made in this paper so as to detect the orientation of the X-ray images for image retrieval that aims for automated annotation. The most relevant Discrete Wavelet Transformation (DWT) coefficients were used as features and the X-rays were classified using Support Vector
Machine (SVM) and Probabilistic Neural Network (PNN). An effort has been made to automatically search and classify the X-ray images into six classes namely chest, spine, foot, palm, head and neck. Each class consists of 30 images. The image dataset represents different ages, genders, views, positions and pathologies. The classification techniques and automatic code generation are very much helpful in teaching, research, diagnostic analysis and hence this method is proposed.

This paper has been organized as: Section I gives an outlay of the general introduction and the need of the proposed work. Review of the existing Literature has been presented in Section II. Section III elaborates the Proposed Methodology, while the Performance Measures and Experimental Results are described in Section IV and V respectively that are followed by the conclusion in Section VI.

\section{LITERATURE REVIEW}

An extensive survey of the existing literatures has been made with a view to realize the on-going trends in the field of automated annotation of X-ray images for efficient retrieval of medical-images, which gives an insight into the issues and problems that are currently faced by the prevailing methodologies and by which their shortcomings can be addressed efficiently. 
Image processing and pattern recognition techniques are practically important to provide the required information in diagnosis and treatment for medical imaging. The progress in these techniques is reflected in the sophisticated software tools, some of which are commercially available, and others may still be in research and development stage [1]. The M3 Filter is proposed which is a hybridization of mean and median filter. It replaces the central pixel by the maximum value of mean and median for each sub images [2]. The Paper in [3] presents with two different texture features namely 32 bin gray scale histogram and statistical features, which provide information about the properties of the level of the intensity distribution in the echo cardiogram image. EM algorithm is used to find the most likely distribution function to describe the relation between obvious variables and latent variables has been presented in [4]. A segmentation algorithm based on the kernelized weighted Cmeans clustering and automatic segmentation correctness coefficients is proposed in [5]. The Paper in [6] describes the removal of the noise and proposes with the enhancement in terms of the contrast using high boost filter and Laplacian of Gaussian filter. An EM algorithm for segmentation and powerful probabilistic modeling tool that are used to provide a model-based clustering of Transform domain features namely DWT and DCT coefficients for classification of Xrays have been studied in the work of [7] and [8] respectively. The work in [9] attempts to recognize the various views by image matching that employs a set of local key points as features. A generalized approach to histogram matching is given in [10]. An automatic classification of cardiac views in Echocardiogram using BPNN and SVM classifier is described in paper [11]. The author in [12] describes the Decision tree classifier to address the problem of automatic image annotation for medical image retrieval. The work in [14] describes the Classification of X-rays using Statistical Moments and SVM.

\section{PROPOSED METHODOLOGY}

In this system, the proposed work consists of three main stages namely image pre-processing, feature extraction and view classification that have been used in order to classify the views of the X-ray images.

Medical X-ray images are taken as input to the system. M3 filter is applied on the input image to reduce the noise and to improve the contrast. Filtered images have been segmented using Expectation Maximization (EM) algorithm. From the segmented images, the features have been extracted using Discrete Wavelet Transform (DWT). SVM classifier, PNN classifier and Decision tree algorithm are used for image classification process to classify the X-ray images of head, foot, palm, chest, neck and spine. The block diagram of the proposed method is shown in Fig. 1.

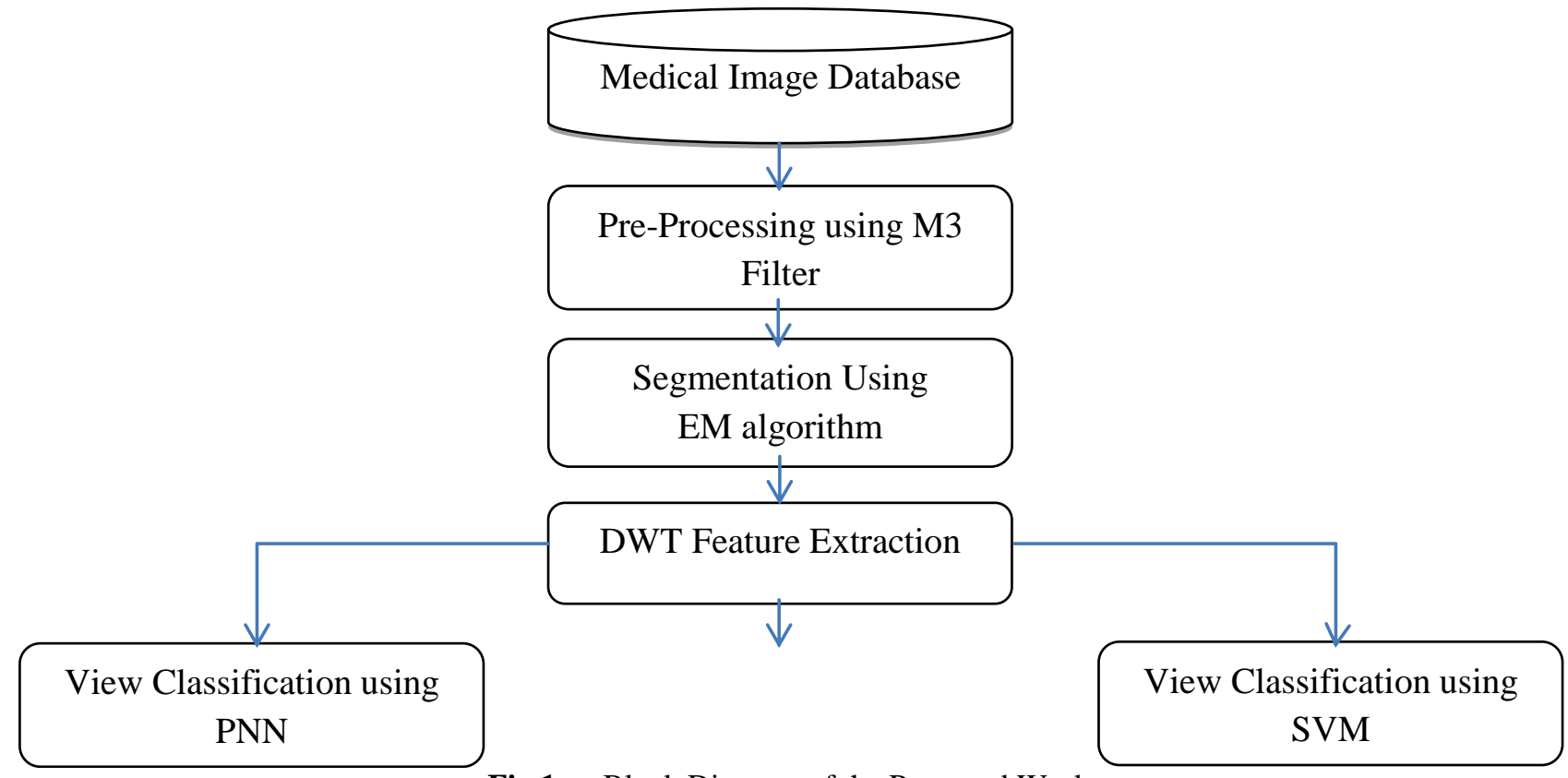

Fig.1 Block Diagram of the Proposed Work

\subsection{Data Source}

The X-ray images used extensively in this work have been obtained from the following

(i) IRMA database of the Department of Diagnostic Radiology, Aachen, Germany and

(ii) Department of Radiology of the Raja Muthaiah Medical College and Hospital, Annamalai University.

\subsection{Pre-Processing}

In medical image processing, Pre-processing is mandatorily to be done at inception so as to increase the reliability of the optical inspection of the images, which can usually be done by a variety of filters that aim at enhancement or augmentation of the quality of images with a view to allow room towards the further stages of processing. In this paper, M3 filter has been incorporated for the pre-processing of images, which is basically a combination of mean and 
median filter, which performs far better than that of the others in removing the noise by replacing the maximum value of mean and median in the central pixel for each sub images.

\subsection{Segmentation}

The success of image analysis depends on reliability of segmentation, but an accurate partitioning of an image is generally a very challenging problem. The goal is to simplify the representation of the images and to find the Region of Interest (RoI) that is found by applying EM algorithm, which computes the estimation of maximum likelihood of unknown parameters with the assumed parameters.

The EM method is iterative that assumes the parameters initially. Then, by using the feature value of the images, it computes the mean, variance, probability density function and normalized probability values in E-step. The new mean and variance are calculated by using the above calculated values in M-step. These steps are repeated until the model resembles the behaviour described by the samples.

\subsection{Feature Extraction}

Feature extraction is a technique which is used to extract and represent the contents of the X-ray images. In this work, features are extracted using Discrete Wavelet Transform (DWT). Wavelet decomposition is the first step in feature extraction. This operation returns the wavelet decomposition of the image at predefined scale using the wavelet. The decomposition vector consists of one approximation coefficient vector and three detail coefficient vector namely, horizontal detail coefficients, vertical detail coefficients and diagonal detail coefficients. In this work, vertical, horizontal and approximate coefficient vectors have been taken upto four levels using Haar coefficients.

\subsection{View Classification}

For the proposed method, the different views that are taken into account are AP, lateral and oblique views, wherein for the set of images of chest, skull, spine and neck, the AP and lateral views are considered, while the oblique and AP are extended towards the images of the foot and palm. The aforesaid views of the X-ray images are detected using PNN, decision tree algorithm and SVM. The three different views so mentioned above are given in Fig. 2 (a) to (c) for an example class of Chest X-ray images.

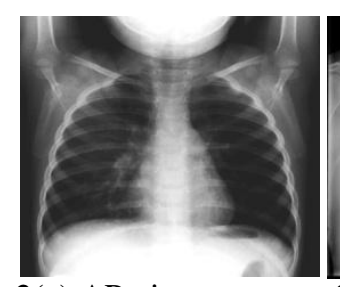

2(a) AP view

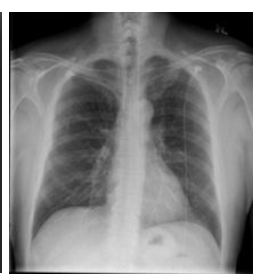

2(b) PA view

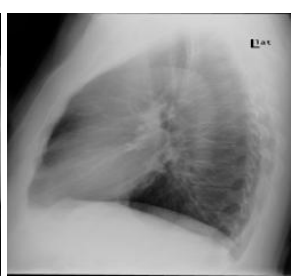

2(c) Lateral view
Fig.2 Views of X-ray images
The PNN is a feed forward neural network that implements the Bayesian decision strategy for classifying input vector. It consists of four layers namely Input layer, Pattern layer, Summation layer and Output layer. PNN is often used in classification problems. The architecture of PNN is shown in Fig. 3.

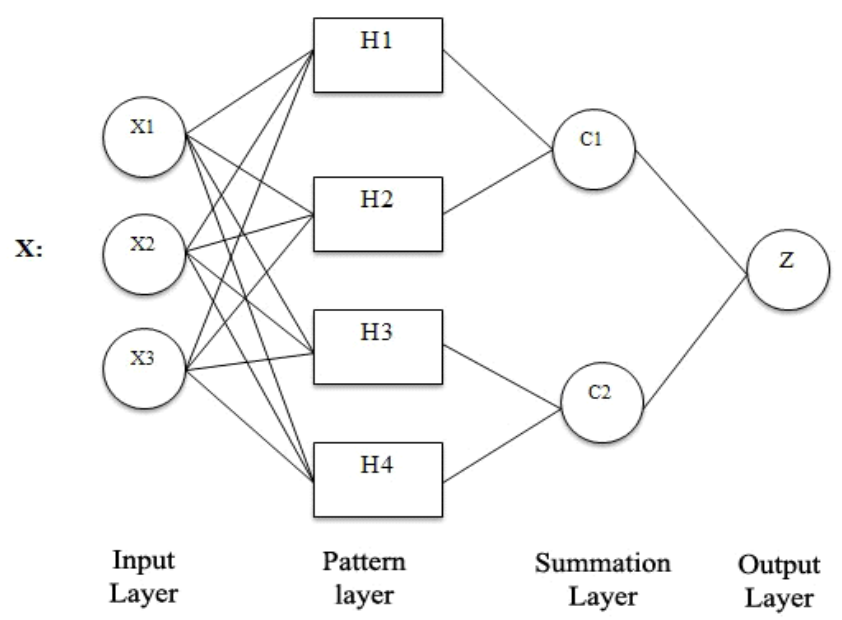

Fig.3 Architectureof PNN

In this work, input layer is used to represent the input pattern or feature vector, which is fully inter-connected with the hidden layer that consists of the training data set. The actual example vector serves as the weights as applied to the input layer. In the summation layer the product of two vectors from the hidden layer is summated to find its average value. Finally, an output layer represents each of the possible classes for which the input data can be classified. In this work, the feature vectors ' $F$ ' are taken as input and its mean and variance ' $\sigma$ 'are computed to calculate weight ' $\mathrm{w}$ '

$$
w=e\left|\frac{x-x_{11}}{\sigma}\right|^{2}
$$

And this weight is multiplied with the input vector ' $F$ ' in the hidden layer.

$$
H=W_{i} F
$$

In summation layer ' $C_{j}$ ', the product of two vectors from the hidden layers are summated and its average value is computed.

$$
c_{j}=\frac{\sum_{i=1}^{N} e^{\frac{\left(h_{i-1}\right)}{\gamma^{2}}}}{N}
$$

The maximum of the average value is compared with the values of the test image in the output layer. If the estimated value is similar with the test value in the output layer, the images are correctly classified, else it is mis-classified.

\subsection{Decision Tree}

Decision tree algorithm is a formal, structured approach to making decision based on the rules. It helps to decompose a complex problem into smaller and manageable understanding. It is also non-parametric, because for each class the distribution of the variable does not require any assumptions. It consists of root node represented by 'T',

\subsection{Probabilistic Neural Network}


Chance node or leaf node as ' $\mathrm{C}$ ', and terminal node as ' $\mathrm{T}$ '. Each class contains decision criteria based on one feature. Features with close resemblance are used for the first split and this procedure is repeated until there is no further split. Decision tree is constructed from the training dataset, which consists of objects described by the set of attributes and class label. The class that is associated with leaf is the output of the tree. The tree misclassifies the images, when the output of the tree does not match with the class label. The structure of the decision tree is shown in Fig. 4.

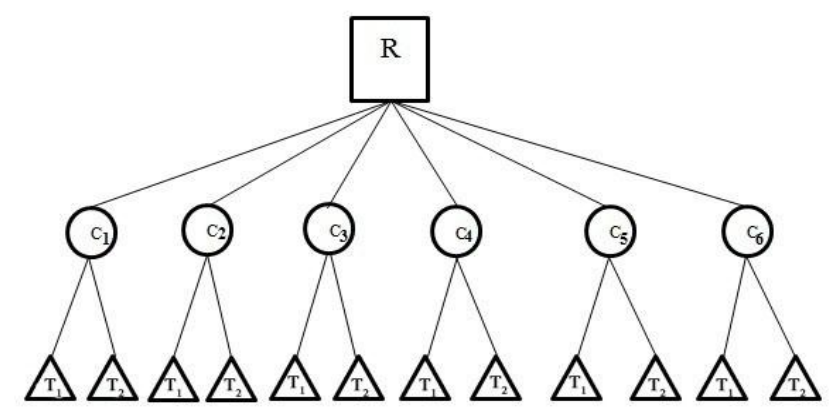

Fig.4 Architecture of Decision Tree

In this work, six chance nodes ' $\boldsymbol{C}_{\mathbf{1}}$ ' to ' $\boldsymbol{C}_{\mathbf{6}}$ ' represents the six different classes of X-ray images namely chest, foot, palm, skull, hand and spine based on the similarity measures using City block distance.

$$
D=x_{1}-x_{2}+y_{1}-y_{2} \text {, here } x_{1}, x_{2} \text { is test image }
$$

and $y_{1}, y_{2}$ is database image.

The probability will be assigned to each branch emanating from each chance node. Based on the probability, the two terminal nodes $T_{1}$ and $T_{2}$ for each class are assigned for the anterior view and lateral view. If the probability is greater than 0.6 then the orientation of the X-ray images is said to be anterior view else it is said to be of lateral or oblique view.

\subsection{Support Vector Machines}

Support Vector Machine is a supervised machine learning algorithm that uses kernel function to map linearly inseparable data to linearly separable data by mapping given data in higher dimension. A hyperplane is constructed in such a way that the margin between the two classes is maximum. The data vectors lying near the hyperplane are called support vectors which are alone then classified rather than considering all data points unlike clustering algorithms. In this work, classification is employed in classifying two categories namely anterior view and lateral view for chest, skull, neck and spine, whereas AP and oblique for foot and palm of X-ray images, in which two binary SVM classifiers are created and trained to distinguish one class from the other.

\section{PERFORMANCE MEASURES}

Several performance metrices are measured for the results of classification such as sensitivity, specificity and accuracy. The quality of classification is measured from a confusion matrix which records correctly and incorrectly recognized examples for each class. The actual and predicted cases produced by classification system can be provided by a confusion matrix and it is given in Table 1. TP is the number of true positives, FP is the number of false positives, TN is the number of true negatives and $\mathrm{FN}$ is the number of false negatives. Accuracy measures the quality of the classification by finding the true and false positives and true and false negatives. Whereas sensitivity deals with only positive cases and specificity deals with only negative cases.

TABLE 1 Confusion matrix on performance indices

\begin{tabular}{|l|l|l|}
\hline \multirow{2}{*}{ Actual } & Predicted \\
\cline { 2 - 3 } & Positive & Negative \\
\hline Positive & True positive & False positive \\
\hline Negative & False Negative & True Negative \\
\hline
\end{tabular}

The performance measures sensitivity, specificity, and accuracy which are calculated as follows:

$$
\begin{aligned}
& \text { Accuracy }=\frac{t p+t n}{t p+t n+f p+f n} \\
& \text { Sensitivity }=\frac{t p}{t p+f n} \\
& \text { Specificity }=\frac{t n}{t n+f p}
\end{aligned}
$$

The specificity and sensitivity are the two most important characteristics in medical image analysis. Sensitivity deals with only positive cases and specificity deals with only negative cases, while the quality of the classification is measured by using accuracy. Accuracy is generally regarded with balanced measure whereas sensitivity deals with only positive cases and specificity deals with only negative cases.

\section{EXPERIMENTAL RESULT}

For the experimental process, six classes of X-ray images namely chest, foot, palm, skull, neck and spine of each of 30 images have been considered. Initially, the X-ray images are preprocessed and segmented. The sample result of preprocessed and segmented X-ray images is shown in Fig. 5(a) and Fig. 5(b). Features are extracted from the segmented images and the extracted features are used to test the X-ray images for the classification using PNN classifier, Decision Tree and SVM classifier. In this paper, the SVM with DWT features provides better accuracy than that of PNN classifier and Decision tree algorithm. The result of the performance measures for the PNN classifier, Decision Tree Algorithm and SVM classifier is shown in Table 2, Table 3, and Table 4 and the comparison between these three classifiers for its performance is given in the Table 5 .

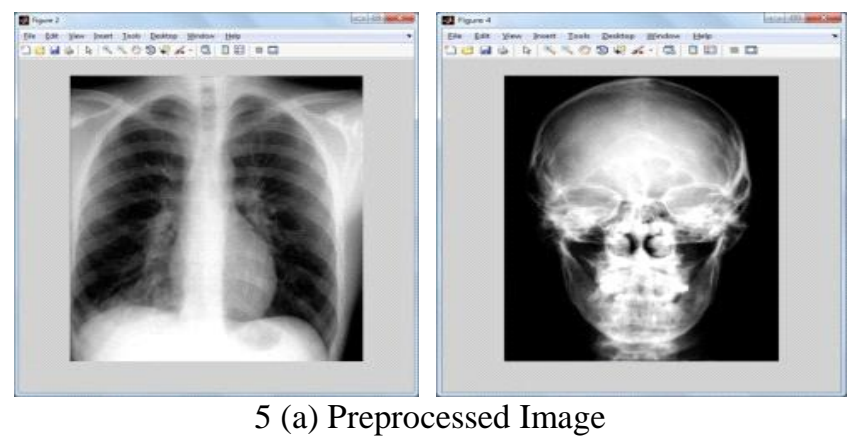

5 (a) Preprocessed Image 


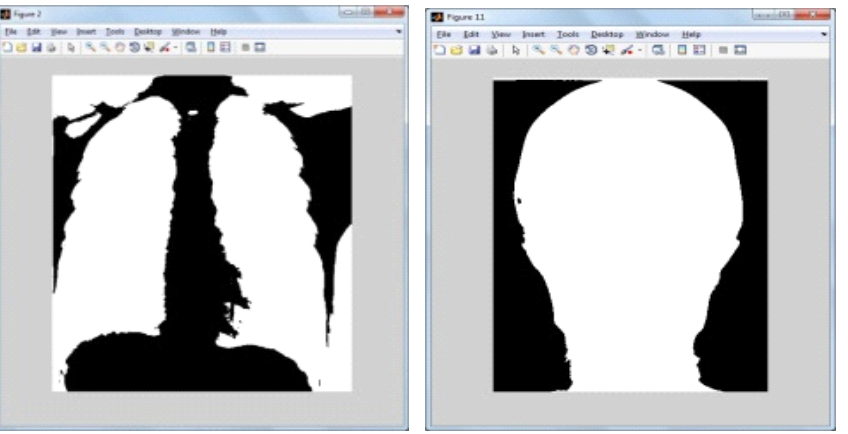

5(b) Segmented Image

Fig.5 Result of Preprocessed and Segmented X-ray Images

TABLE 2 Performance measure for PNN

\begin{tabular}{|l|l|l|l|}
\hline X-ray Image & $\begin{array}{l}\text { Accuracy } \\
(\boldsymbol{\%})\end{array}$ & $\begin{array}{l}\text { Sensitivity } \\
(\boldsymbol{\%})\end{array}$ & $\begin{array}{l}\text { Specificity } \\
(\boldsymbol{\%})\end{array}$ \\
\hline Chest & 85.71 & 71.43 & 100 \\
\hline Foot & 71.43 & 57.14 & 85.71 \\
\hline Palm & 64.29 & 57.14 & 71.43 \\
\hline Skull & 78.57 & 85.71 & 71.43 \\
\hline Spine & 78.57 & 71.43 & 85.71 \\
\hline Neck & 71.43 & 71.43 & 71.43 \\
\hline $\begin{array}{l}\text { Overall } \\
\text { Performance }\end{array}$ & 75 & 69.04 & 80.95 \\
\hline
\end{tabular}

TABLE 3 Performance measure of Decision Tree

\begin{tabular}{|l|l|l|l|}
\hline X-ray Image & $\begin{array}{l}\text { Accuracy } \\
(\boldsymbol{\%})\end{array}$ & $\begin{array}{l}\text { Sensitivity } \\
(\mathbf{\%})\end{array}$ & $\begin{array}{l}\text { Specificity } \\
(\mathbf{\%})\end{array}$ \\
\hline Chest & 93.33 & 80 & 96 \\
\hline Foot & 88.89 & 73.33 & 92 \\
\hline Palm & 95.56 & 86.67 & 97.33 \\
\hline Skull & 96.67 & 86.67 & 98.67 \\
\hline Spine & 90 & 80 & 92 \\
\hline Neck & 92.22 & 66.67 & 97.33 \\
\hline $\begin{array}{l}\text { Overall } \\
\text { Performance }\end{array}$ & 92.77 & 78.89 & 95.55 \\
\hline
\end{tabular}

TABLE 4 Performance measure of SVM

\begin{tabular}{|l|l|l|l|}
\hline X-ray Image & $\begin{array}{l}\text { Accuracy } \\
(\boldsymbol{\%})\end{array}$ & $\begin{array}{l}\text { Sensitivity } \\
(\boldsymbol{\%})\end{array}$ & $\begin{array}{l}\text { Specificity } \\
(\boldsymbol{\%})\end{array}$ \\
\hline Chest & 96.67 & 100 & 93.33 \\
\hline Foot & 90 & 100 & 80 \\
\hline Palm & 93.33 & 93.33 & 93.33 \\
\hline Skull & 96.67 & 93.33 & 100 \\
\hline Spine & 90 & 80 & 100 \\
\hline Neck & 93.33 & 86.67 & 100 \\
\hline $\begin{array}{l}\text { Overall } \\
\text { Performance }\end{array}$ & 93.33 & 92.22 & 94.44 \\
\hline
\end{tabular}

TABLE 5 Comparison between PNN, SVM and Decision Tree

\begin{tabular}{|l|l|l|l|}
\hline Classifier & $\begin{array}{l}\text { Accuracy } \\
(\%)\end{array}$ & $\begin{array}{l}\text { Sensitivity } \\
(\%)\end{array}$ & $\begin{array}{l}\text { Specificity } \\
(\%)\end{array}$ \\
\hline PNN & 75 & 69.04 & 80.95 \\
\hline SVM & 93.33 & 92.22 & 94.44 \\
\hline $\begin{array}{l}\text { Decision } \\
\text { Tree }\end{array}$ & 92.77 & 78.89 & 95.55 \\
\hline
\end{tabular}

\section{CONCLUSION}

In this paper, view classification of medical X-ray images are automated by extracting DWT features. PNN classifier, Decision Tree Algorithm and SVM classifier were used to test the usefulness of the features in correctly classifying the $\mathrm{X}$-ray views. The result obtained for the five classes of Xray images are promising as obtained through SVM in terms of accuracy for chest, foot, spine, neck and skull, whereas for the palm, the accuracy yielded by Decision tree algorithm has been proven better. Thus, the attempt has been accomplished in this paper to bring out the view classification for the different classes of X-ray images by a couple of efficient classifiers, yet with very high accuracy.

\section{REFERENCE}

[1]. ShantalaPatill and Dr.KairanKumariPatil. "Roles of Computer Vision, Image Processing and Pattern Recognition in Health Application." CSI Communications, January 2014.

[2]. SumathiGanesanT.S.SubashiniE.Pavendhan,

"Automated Annotation Of X-Ray Images Using Statistical Moment Features." (IJAER),Volume 9, Number 20 (2014) Special Issues, Pp.4395-4400.

[3]. Roy A, Sural S, Mukherjee J, Majumdar AK. Statebased modeling and object extraction from echocardiogram video. Information Technology in Biomedicine, IEEE Transactions on. IEEE; 2008;12(3):366-376.

[4]. Mohamed Ali Mahjoub1 KarimKalti 2 "Image segmentation by Adaptive Distance Based on EM algorithm"(IJACSA) International Journal of Advanced Computer Science and Applications, Special Issue on Image processing and Analysis May 2011.

[5]. M. Bugdol, J. Czajkowska, and E. Pietka, "A novel model-based approach to left ventricle segmentation," in Computing in Cardiology (CinC). Krakow, Poland: IEEE, 2012, pp. 561-564.

[6]. Balaji G.N, Subashini T.S, and Chidambaram N.,"Detection of Heart Muscle Damage from Automated Analysis of Echocardiogram video", IETE Journal of Research, February 2015.

[7]. K. Vaidehi, T.S.Subashini, V. Ramalingam, S. Palanivel, M. Kalaimani, "Transform based approaches for Palmprint Identification", International Journal of Computer Applications, Vol. 41, No. 1, 2012

[8]. SumathiGanesan and T.S. Subashini, “An Approach toward the Efficient Indexing and Retrieval on Medical X-ray Images", International Journal of Computer Applications, Vol. 76, No. 12, PP: 7-10 2013.

[9]. Lowe, David G. "Distinctive image features from scale-invariant keypoints." International journal of computer vision 60, no. 2 (2004): 91-110.

[10]. Schiele B, Crowley JL. Recognition without correspondence using multidimensional receptive field histograms. International Journal of Computer Vision. Springer; 2000;36(1):31-50. 
[11]. Balaji G.N, Subashini T.S, and Chidambaram N, "Automatic classification of Cardiac Views in Echocardiogram using Histogram and Statistical Features" , ICICT 2014, ELSEVIER, Procedia Computer Science.

[12]. Wei Li and Maosong Sun, "Automatic Image Annotation Using Maximum Entropy Model", IJCNLP 2005, LNAI 3651,pp. 34-45, 2005 SpringerVerlag Berlin Heidelberg.

[13]. Dahab, Dina Aboul, Samy SA Ghoniemy, and Gamal M. Selim. "Automated brain tumor detection and identification using image processing and probabilistic neural network techniques." International Journal of Image Processing and Visual Communication 1, no. 2 (2012): 1-8.

[14]. Ganesan, Sumathi, T. S. Subashini, and K. Jayalakshmi. "Classification of X-rays using statistical moments and SVM." In Communications and Signal Processing (ICCSP), 2014 International Conference on, pp. 1109-1112. IEEE, 2014.

[15]. http://www.imageclef.org/ImageCLEF2008 